\title{
Three-Directional Reconstruction of a Massive Perineal Defect after Wide Local Excision of Extramammary Paget's Disease
}

\author{
Seung Woo Cho, Hak Chang, Sung Tack Kwon \\ Departments of Plastic and Reconstructive Surgery, Seoul National University College of Medicine, Seoul, Korea
}

Extramammary Paget's disease (EMPD) is a rare, slow-growing intraepithelial malignancy that mainly involves the genital region, including the vulva, penis, scrotum, perianal, and periurethral areas. Although several treatment options exist, wide local excision with a safe margin is considered the treatment of choice. After resection of the lesion, it is often challenging to reconstruct the defect because defects in the perineal region require adequate volume for protection and are susceptible to infections, which is a particularly significant risk for large defects. We report a case of perivulvar EMPD that was reconstructed with three-directional local flaps after wide excision of the tumor. We covered the defect sequentially using the following 3 flaps: a gracilis myocutaneous flap from the left thigh, a bipedicled V-Y advancement flap from the lower abdomen, and an internal pudendal artery perforator-based island flap from the right buttock. To the best of our knowledge, this report is the first to describe a three-directional approach to extensive perivulvar reconstruction.

Keywords Myocutaneous flaps / Perforator flaps / Vulva
Correspondence: Hak Chang Department of Plastic and Reconstructive Surgery, Seoul National University College of Medicine, 103 Daehak-ro, Jongno-gu, Seoul 03080, Korea

Tel: +82-2-2072-3086

Fax: +82-2-747-5130

E-mail: hchang@snu.ac.kr

No potential conflict of interest relevant to this article was reported.

\section{INTRODUCTION}

Extramammary Paget's disease (EMPD) is a rare, slow-growing intraepithelial malignancy that mainly involves the genital region, including the vulva, penis, scrotum, perianal, and periurethral areas, and less commonly the axilla, in elderly patients [1]. Since it is difficult to distinguish from other skin lesions clinically, it is often misdiagnosed as eczema, contact or irritant dermatitis, seborrheic dermatitis, or Bowen's disease. Although EMPD is a slow-growing malignancy, regional and distant metastasis through dermal invasion or lymphatic spread have been reported $[1]$.

Many therapeutic modalities for EMPD have been suggested, including $5 \%$ imiquimoid topical cream, radiotherapy, laser treatment, and modified Mohs surgery. However, wide local excision with a safe margin is considered the treatment of choice. The optimal resection margin is difficult to determine, and we have therefore developed an algorithm using mapping biopsies [2]

After appropriate resection of the lesion, the next step is reconstruction of the defects. This is often challenging because the perineal region requires adequate volume for protection and is susceptible to infection. Skin grafting is vulnerable to trauma and infection, and some local flaps have insufficient volume or are difficult to fit into place given the size of the defect. Gracilis myocutaneous flaps are widely used for perivulvar reconstruction. However, they are not always sufficient for defects larger 
than $15 \times 10 \mathrm{~cm}[3]$.

Here, we report a case of perivulvar EMPD that was reconstructed with three-directional local flaps after wide excision of the tumor. To the best of our knowledge, this report is the first to describe a three-directional approach to perivulvar reconstruction.

\section{CASE}

A 68-year-old female with a history of diabetes and hypertension was referred to the Plastic and Reconstructive Surgery Department for the evaluation and further treatment of erythematous scaly plaques on the perivulvar area that had shown a tendency to bleed for approximately 10 years. She had received treatment at another outpatient department, but results were not successful. The patient complained of mild pain, itching, and bloody discharge at the lesion (Fig. 1). A biopsy was performed by a dermatologist on September 11, 2015 and Paget's disease was diagnosed. Preoperative imaging, including a pelvic computed tomography scan, pelvic ultrasonography, and a wholebody fluorodeoxyglucose positron emission tomography scan, showed no evidence of distant metastasis. One month after the first visit, a mapping biopsy was performed while the skin was unfolded by gentle traction according to the previously described protocol used in our clinic (Fig. 2) [2]. The mapping biopsy results were positive at some sites involving the periurethral area, and we therefore referred the patient to a gynecologist for consultation. We decided to perform frozen biopsies at several sites at the operation room, including the periurethral area, instead of an additional mapping biopsy, in order to minimize unexpected damage to the urethra. After 6 weeks, a wide excision

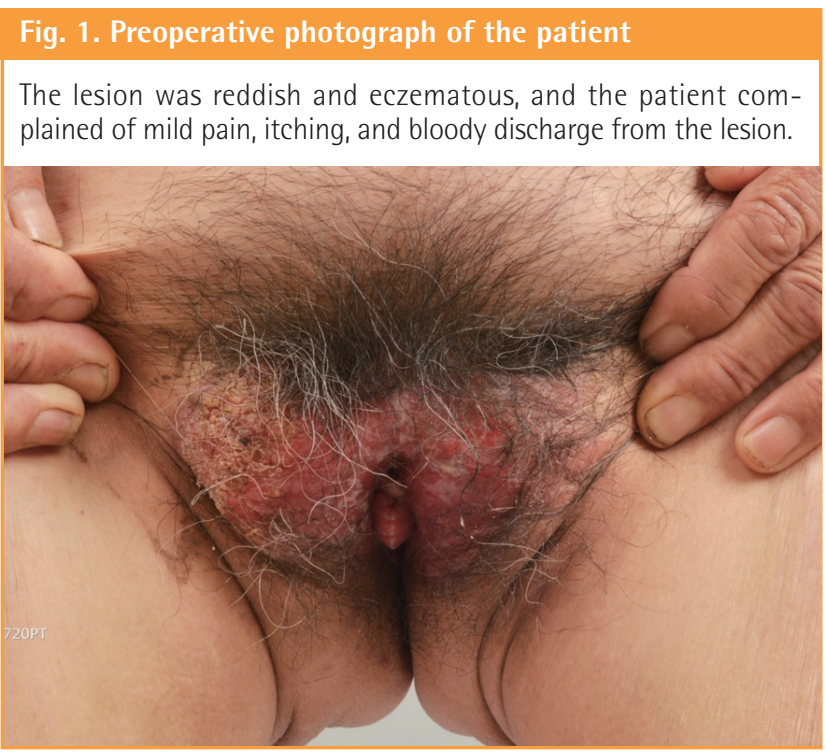

of the tumor with a $2 \mathrm{~cm}$ safety margin was performed (Fig. 3A). After it was proven by frozen biopsies that no malignancy was present in the periurethral area or tumor margin, the defect, which was $14.5 \times 15.0 \mathrm{~cm}$ in size, was reconstructed with threedirectional local flaps. First, we designed a gracilis myocutaneous flap on the left thigh. We approached the gracilis muscle, the muscle was cut distally, and then was rotated to the perivulvar area to cover the defect (Fig. 3B) [3]. Second, after marking the range covered by the first flap, we designed a bipedicled V-Y advancement flap from the lower abdomen to cover the upper side of the defect. We dissected subcutaneously, preserving the perforators of the flap. Next, the remaining defect was covered with a right internal pudendal artery perforator-based island flap as previously described (Fig. 3C, D) [4]. The specimen was sent to a pathologist and demonstrated to be EMPD (Fig. 4). The wound healed without any problems, and all stitches were removed after 3 weeks. No local recurrence has been observed. The patient reported no discomfort at the donor site or in the perineal area at a 5-month follow-up (Fig. 5).

\section{DISCUSSION}

In 1874, Paget [5] first described an intraepidermal neoplasm of the nipple and areola, often preceded by breast cancer. It usually occurs in areas rich in apocrine glands, such as the penis, scrotum, vulva, perineum, and axillae of middle-aged and older individuals and presents as reddish patches or gray plaques that are eczematoid or papillomatous [6]. Although EMPD is an intraepidermal neoplasm, it is often related to malignancies of the lower gastrointestinal or genitourinary tract, and dermal invasion and lymph node metastasis have been reported. Many con-

\section{Fig. 2. Mapping biopsy of the patient}

Punch biopsies were performed at the margin of the lesion and 2 $\mathrm{cm}$ away from the lesion.

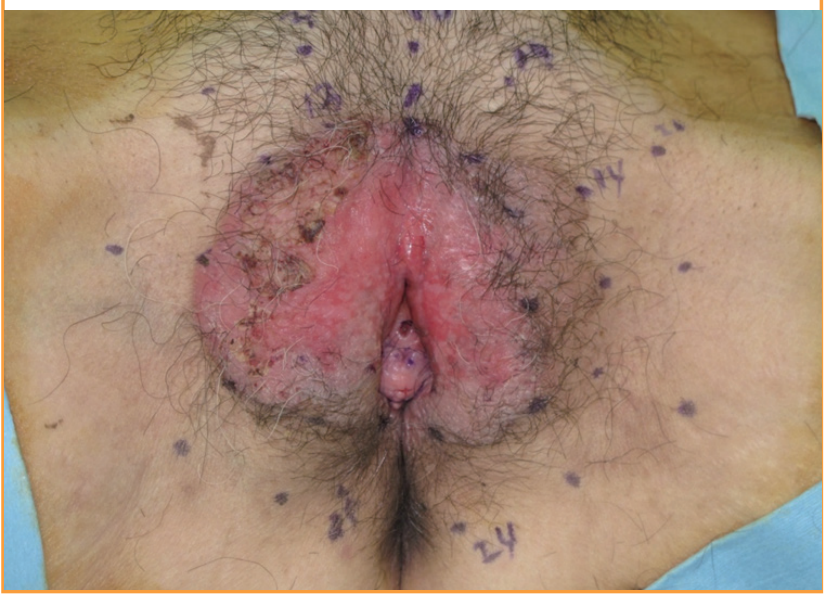




\section{Fig. 3. Surgical procedures involved in the three-directional reconstruction}

(A) A perivulvar defect measuring $14.5 \times 15.0 \mathrm{~cm}$ was observed. (B) A pedicled gracilis myocutaneous flap was designed and the distal portion of the gracilis muscle was cut. (C) A pedicled internal pudendal artery perforator-based island flap was designed. (D) A postoperative photograph was taken after three-directional reconstruction was completed.
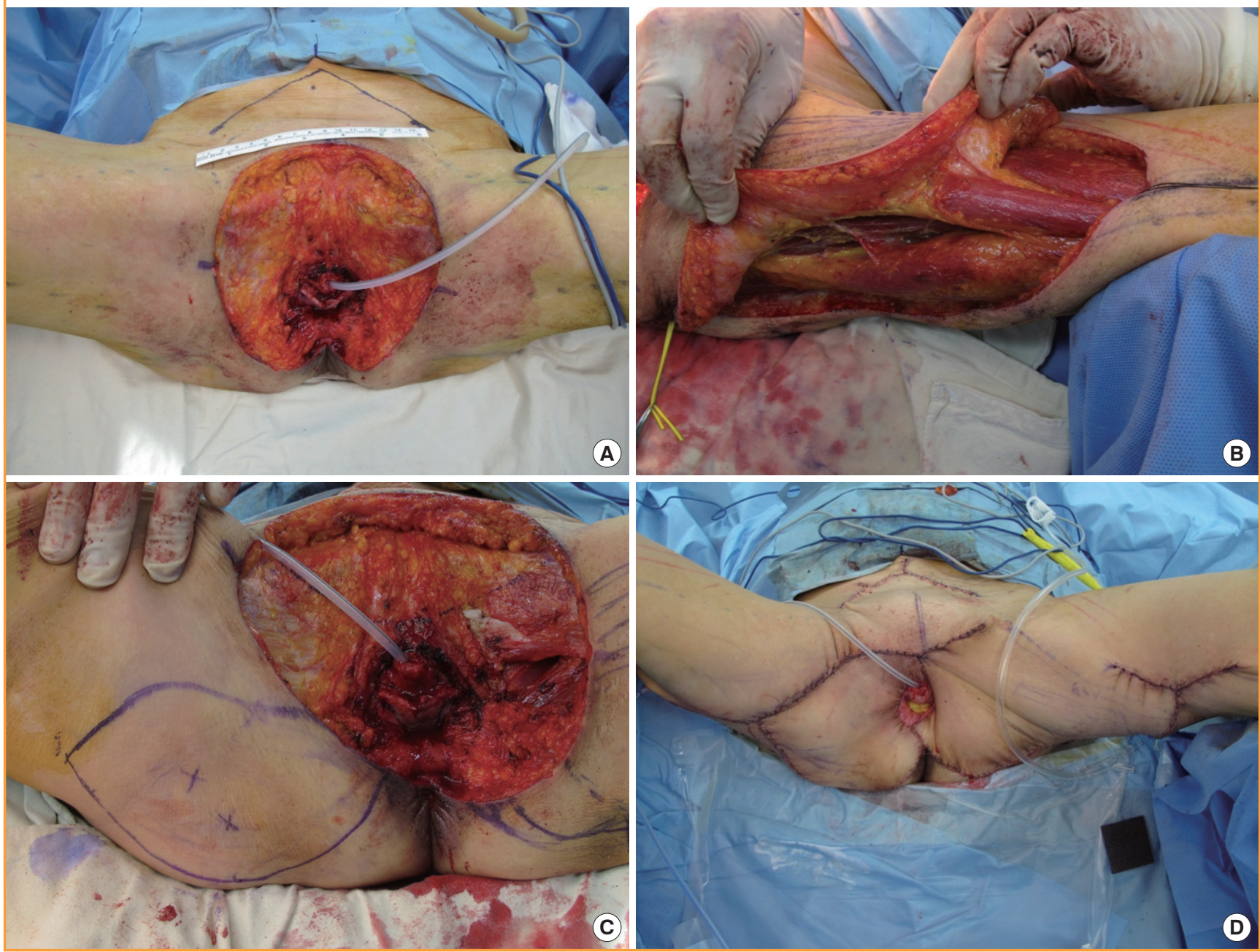

Fig. 4. Pathological specimen of this case, showing EMPD

The biopsy specimen presents clusters of single cells (black arrow) within the epidermis that have pleomorphic nuclei with prominent nucleoli and pale-staining abundant cytoplasm $\left(H \& E_{1} \times 400\right)$. EMPD, extramammary Paget's disease.

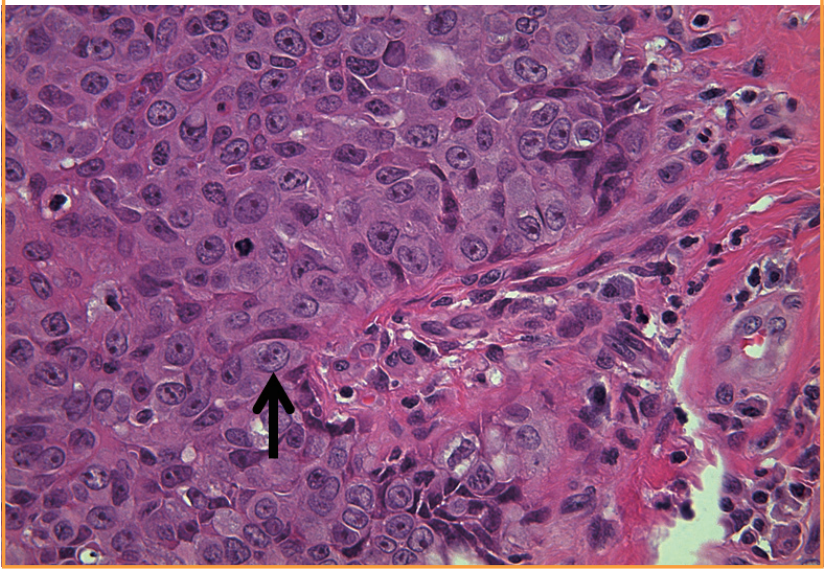

Fig. 5. Photograph taken 5 months postoperatively

The flaps were well-maintained 5 months after the operation. The patient reported no limitations wearing clothes or walking.




servative treatments have been introduced for EMPD, but controversy remains regarding their effectiveness. Hence, complete resection is recommended for the definitive treatment of EMPD.

Perineal defects following the wide local excision of EMPD are difficult to reconstruct in a manner that results in favorable functional and cosmetic outcomes. Coverage of extensive defects in this area requires more than a simple skin graft. When extensive soft tissue defects occur as a result of the wide local excision of a neoplasm, the cosmetic outcomes are less satisfying, and direct skin grafting may result in poor soft tissue padding for protection of the region. Moreover, many bacteria are present in and around the vagina and anus, including Lactobacillus, Bacteroides fragilis, and Escherichia coli, and grafted skin can be easily contaminated by such bacteria, resulting in gradual loss and contraction [7]. Finally, contraction may occur when grafted skin heals poorly, resulting in skin maceration and breakdown.

Many flaps have been introduced to reconstruct extensive defects around the vulva, scrotum, penis, and scrotum instead of skin grafts, including myocutaneous flaps such as a unilateral gracilis flap, a tensor fascia latae flap, and a rectus abdominis flap $[8,9]$. These flaps provide good coverage for perineal defects and are associated with satisfactory functional outcomes. However, one local flap is not sufficient to cover very large defects. Moreover, the blood supply may become unreliable even if the defect is covered with a large amount of advancement or rotation. Eventually, an insufficient blood supply causes flap necrosis. For very large defects, bilateral gracilis myocutaneous flaps are widely used. However, if the defect is larger than $15 \times 10 \mathrm{~cm}$, it cannot be covered using only a bidirectional approach [3]. Even if it is covered with bilateral gracilis myocutaneous flaps, some patients treated at our institution have complained of tension around the perineum when walking.

Fasciocutaneous flaps were widely used in the past [7]. They provided a pliable local flap and good protection for superficial defects. However, fasciocutaneous flaps lack soft tissue volume and mobility, and therefore they lead to less favorable outcomes, such as skin grafting.

Free flaps are also a reconstructive option for massive perineal defects. However, they pose technical challenges as well as requiring more sophisticated postoperative care and a longer operation time. Specifically, the free transverse rectus abdominis musculocutaneous flap can be considered as an option [10]. However, this flap tends to be too bulky for perineal reconstruction and it requires delicate postoperative care and a longer operation time. Few reports have been published regarding free flaps for perineal reconstruction [8].

In this case, we reconstructed an extensive perineal defect with three-directional local flaps. This approach has some advantag- es. First, it provides sufficient soft tissue padding and range of motion. In contrast, the use of skin grafting to treat such a massive defect can lead to severe contraction and loss of volume. Additionally, using this approach, an extensive defect that cannot be covered with one local flap can be adequately reconstructed with excellent cosmetic and functional outcomes. This defect, which measured $14.5 \times 15.0 \mathrm{~cm}$, was too large to be covered with one or two local flaps. Second, as is the case for other myocutaneous flaps, three-directional flaps are resistant to infections. As discussed above, since many bacteria are present around the vagina and anus, grafted skin or raw surfaces can easily be contaminated. Third, a delicate design can be achieved, as three sequential flaps are used in this approach. The deeper and more bulky portion of the defect can be covered with a pedicled gracilis myocutaneous flap, while two other flaps are used to cover the thinner and shallower portion. Moreover, since the flaps are applied sequentially, the size of the flaps can be adjusted in order to minimize waste of the materials. However, this approach also has some shortcomings. The design is quite sophisticated and the operation time is longer than is required for simple skin grafting.

In conclusion, three-directional reconstruction of an extensive perineal defect can provide delicate restoration of the shape and successful protection, minimizing donor site morbidity. Therefore, three-directional reconstruction may be an excellent option for the reconstruction of very large perineal defects despite the longer operation time.

\section{REFERENCES}

1. Chan JY, Li GK, Chung JH, et al. Extramammary Paget's disease: 20 years of experience in chinese population. Int J Surg Oncol 2012;2012:416418.

2. Kim BJ, Park SK, Chang H. The effectiveness of mapping biopsy in patients with extramammary Paget's disease. Arch Plast Surg 2014;41:753-8.

3. Burke TW, Morris M, Roh MS, et al. Perineal reconstruction using single gracilis myocutaneous flaps. Gynecol Oncol 1995;57:221-5.

4. Hashimoto I, Goishi K, Abe Y, et al. The internal pudendal artery perforator thigh flap: a new freestyle pedicle flap for the ischial region. Plast Reconstr Surg Glob Open 2014;2: e142.

5. Paget J. An address delivered at the opening of the section of surgery. Br Med J 1874;2:221-2.

6. Pitman GH, McCarthy JG, Perzin KH, et al. Extramammary Paget's disease. Plast Reconstr Surg 1982;69:238-44.

7. Lee SH, Rah DK, Lee WJ. Penoscrotal reconstruction with gracilis muscle flap and internal pudendal artery perforator 
flap transposition. Urology 2012;79:1390-4.

8. Hsu H, Lin CM, Sun TB, et al. Unilateral gracilis myofasciocutaneous advancement flap for single stage reconstruction of scrotal and perineal defects. J Plast Reconstr Aesthet Surg 2007;60:1055-9.

9. Nisar PJ, Scott HJ. Myocutaneous flap reconstruction of the pelvis after abdominoperineal excision. Colorectal Dis 2009; 11:806-16.

10. McAllister E, Wells K, Chaet M, et al. Perineal reconstruction after surgical extirpation of pelvic malignancies using the transpelvic transverse rectus abdominal myocutaneous flap. Ann Surg Oncol 1994;1:164-8. 